\title{
Properties of the Magellanic Type Galaxies
}

\author{
S. C. Odewahn \\ Dept. of Astronomy, University of Minnesota, Minneapolis, MN 55455
}

\begin{abstract}
Late-type Magellanic spiral galaxies possess high surface brightness stellar bars whose centers are often offset from the photometric and kinematic disk centers derived from stellar and HI distributions. Since these systems represent the transitional stage between the pure disk and irregular galaxies, and because the spiral arm and bar properties of these systems present many unique aspects, an understanding of their properties will benefit our understanding of the physics underlying the Hubble sequence as well as the mechanisms associated with bar formation. I review detailed photometric and kinematic studies of several important SBm galaxies. Statistical studies using large samples drawn from the Third Reference Catalog of Bright Galaxies support the idea that scenarios invoking the formation of offset bars and/or dominant spiral arms through some tidal interaction mechanism might be attractive, since a common trait among the Magellanic spirals appears to be the presence of a physical neighbor.
\end{abstract}

\section{Introduction}

The closest, brightest and best resolved galaxy in the sky, the Large Magellanic Cloud, is the prototype of the late-type disk systems referred to collectively as Magellanic spiral galaxies. These systems, which are assigned a stage value of $\mathrm{T}=9(\mathrm{Sm})$ in the revised Hubble classification system (de Vaucouleurs 1959), represent the latest disk galaxies displaying vestiges of spiral structure. Displaying signs of rotational symmetry and spiral structure, these galaxies typically possess high surface brightness stellar bars whose centers are often offset from the disk center derived from star counts, HI distribution, or the elliptically-shaped outer optical isophotes. As we approach the latest Hubble stages we find a general increase in optical asymmetry, a property which culminates in the Magellanic type to the point where these galaxies are often referred to as "one-armed spirals". Although systematically lower in mass, mean surface brightness, and integrated absolute magnitude than the earlier Hubble stages (Odewahn 1989), most of these systems do not qualify as dwarf galaxies. Because these galaxies represent the transitional stage between the pure disk and irregular galaxies, an understanding of their photometric and kinematic properties will be essential to a full understanding of the physics underlying the Hubble sequence. Studies of the mechanisms producing spiral structure will benefit from studies of the Sm galaxies, since these galaxies represent the last stage in which this very general phenomenon is observed. Because no bulge or lens component is present, 
photometric and kinematic investigations of the bar properties are simplified. Hence, studies of this extremely interesting morphological stage will provide not only basic data necessary for understanding the Hubble sequence, but will also serve to increase our knowledge of such fundamental topics as the formation and maintenance of spiral arms and bars. In this talk I shall review detailed photometric and kinematic studies of several important SBm galaxies, as well as statistical studies of a variety of properties using large samples drawn from the new Third Reference Catalog of Bright Galaxies (de Vancouleurs et al. 1991, RC3), all of which were carried out in an effort to clarify the general properties of this important type of galaxy.

\section{Photometric Properties}

Broadband $B V R I$ surface photometry of a few representative SBm galaxies was carried out using classical photographic and CCD imagery collected at McDonald and Asiago Observatories. A wide range of mean surface brightness is evident among these galaxies, and the overall $B$-band luminosity is dominated by an exponential disk which consistently contributes roughly $50 \%$ of the total luminosity. A dominant, clumpy spiral arm emanates from one end of a bright stellar bar whose center, often broken by a dark lane, is usually well offset from the optical center of the disk defined by ellipse fits to the outer, low surface brightness isophotes. A variety of methods for modeling the two dimensional luminosity distribution of a bar were experimented with (Odewahn 1991) in order to perform detailed luminosity decompositions. For studies in the Johnson $B$ bandpass it was found that the bar component will contribute $10 \%$ to $20 \%$ to the total luminosity, and the smooth arm component contributes roughly $20 \%$ to $30 \%$ of the total luminosity. These results are dependent on the type of bar luminosity model chosen for the decomposition. The contribution to the total luminosity by the resolved $\mathrm{OB}$ star complexes and HII regions, which were found in abundance throughout the dominant asymmetric arm, ranges from $5 \%$ to $15 \%$. In the cases of NGC 4618 , NGC 2537 , and NGC 4027, it was established that the colors of the arm, disk, and discrete emission components follow well the mean color-color curves established for the integrated color properties of galaxies. It is interesting that the bar colors often deviate from this trend significantly. In particular, the bar in NGC 4618 was found to be systematically much bluer in the $V-I$ and $B-I$ color indices than is implied by the mean color sequences for the integrated colors of galaxies. In some cases, such as in the LMC, the bar appears to be extremely red compared to the surrounding galaxy (Bothun \& Thompson 1988), and hence we may not conclude that the bars are always systematically blue. The fact that the LMC bar is depleted in HII may have something to do with its red color, and the idea that the bars in NGC 4027, NGC 2537 and NGC 4618 have undergone more recent epochs of star formation must be investigated further.

Detailed studies of the bar properties in NGC 4618 confirm past qualitative discussions by de Vaucouleurs \& Freeman (1973) concerning the bars in many SBm galaxies. Using a Fourier component analysis of residuals to ellipse fits to the bar isophotes, it was found that the $3 \theta$ component dominates the Fourier fit, especially in the $I$ band, confirming the suspicion that the bar has a somewhat 
triangular shape. In the cases of NGC 4618, NGC 4027, NGC 2366, and the LMC, the luminosity distribution along the bar major axis (after proper disk subtraction), was found to exhibit an exponential decline, a confirmation of the results for bars in late-type galaxies presented by Elmegreen \& Elmegreen (1985). It should be noted that the latest type studied by the Elmegreen's was Sdm. Multi-wavelength imaging of the NGC 4618 bar was used to study the central luminosity dip near the center of the bar. The depth of this luminosity dip was measured with respect to exponential fits to the $B, V$, and $I$ band major axis profiles of the bar. Using a simple Whitford reddening law, it was determined that this central decease in bar luminosity cannot be accounted for solely by the presence of dust. This may indicate a bimodal distribution in the stellar density distribution near the bar center, a characteristic studied theoretically by Duus \& Freeman (1975). It should be noted that recent CCD observations made at CTIO of NGC 7154 and NGC 3664, currently being analyzed, seem to confirm this interpretation of the central dark lanes in some of the Magellanic galaxy bars. More recently, near-infrared $J H K$ surface photometry of NGC 4618 and NGC 2537 (Odewahn et al. 1992) reveal that the lobed appearance of the bar is due to the occurrence of a discrete region located off of the bar center.

\section{Statistical Properties}

A statistical analysis of the RC3 data (Odewahn 1989) has reconfirmed the continuity of the stage axis of the three dimensional classification volume, and in particular has shown that the Magellanic type galaxies fit smoothly into the Hubble sequence in terms of surface brightness, integrated color, mass (implied maximum rotational velocity), and $\mathrm{HI}$ gas content. The frequencies of bar families as a function of Hubble type are shown in Figure 1.

The frequency of bar formation among Magellanic spirals, in fact the latetype systems in general, is quite high. Of the 246 galaxies having literal types of sufficient quality (m.e. $(\mathrm{T})<1.0$ ) approximately $78 \%$ were of the $B$ variety and $19 \%$ were of the $\mathrm{AB}$ variety. This trend is confirmed by a correlation of bar frequency with $\log V_{\mathrm{m}}$, and hence is not the product of selection effects or biased estimations among the classifiers. An analysis of the most recently compiled apparent axis ratio data, referred to the isophotal level of $\mu_{B}=25.0 \mathrm{mag} / \square^{\prime \prime}$ has shown that these late type systems have intrinsic axis ratios which are drawn from a much wider distribution of $q_{0}$ than is found in the earlier stages. If we model the distribution of intrinsic axis ratios, $q_{0}$, as the sum of two Gaussian components, then the mean $q_{\circ}$ of the small $q$ component $\left(q_{1}\right)$ for the Magellanic spirals is $\left\langle q_{1}\right\rangle=0.35$, confirming the results of older studies which show that these galaxies have significantly thicker disks than the early and intermediate stage spirals. Binney (1981) has suggested that this property might be due to increased disk heating introduced by the mass asymmetry of the SBm systems, in which the offset stellar bar induces a resonant coupling between the $\mathrm{z}$-motions of the stars and the radial forces in the disk. 


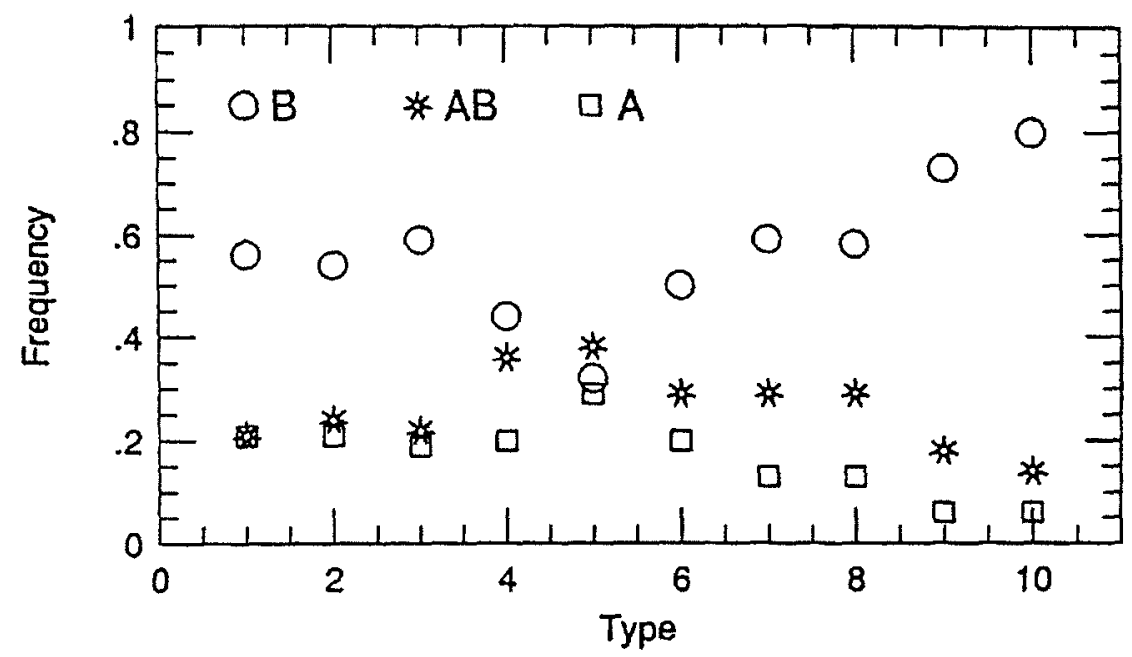

Figure 1. Distribution of RC3 families.

\section{Kinematic Properties}

How does such an apparently asymmetric mass distribution manifest itself in the global velocity field? Is it true, as is implied by the luminosity distribution, that the bar is not centered on the rotational center of the galaxy? This does seem to hold for the case of the LMC (Feitzinger 1980), yet the TAURUS study of NGC 4027 by Pence et al. (1988) found that the bar center is coincident with the rotation center. Kinematic studies of a few Magellanic galaxies utilizing the technique of Fabry-Perot interferometry were undertaken (Odewahn 1989) in order to clarify this question. The velocity fields reveal significant regions of noncircular motion but are clearly dominated by the velocity gradient imposed by rotation. A number of iterative methods were applied to the observed velocity field maps in order to determine the spatial orientation of each galaxy. Studies of NGC 4618, NGC 2537 and LMC show clearly that within the estimated observational uncertainties, the optical center of the stellar bar is displaced by as much as $1.5 \mathrm{kpc}$ from the rotation center and hence the presumed mass center. Using the kinematic center and position angle of the line of nodes, the systemic velocity and the disk inclination derived from photometry, mean rotation curves were computed for each galaxy. These curves are characterized by a large linear region due to solid body rotation (which generally contains the endpoints of the offset bar) and a uniform section in the outer radii. It should be noted that a large asymmetry often exists between the two outer halves of the folded rotation curves, and this seems to be a kinematic trait of the Magellanic type galaxies. The rotation curve was used to construct a best fit axisymmetric velocity field, which was then subtracted from the observed velocity field. Large regions of noncircular or nonuniform motion were evident in the area of the bar endpoints in all five galaxies studied. These were qualitatively attributed to gas streaming 
motions associated with the nonaxisymmetric potential field of the bar, but no clear determination of the types of orbits involved (simple direct oval orbits, etc.) has yet been made. The problem is complicated by the fact that the bar center is displaced from the rotation center, so that transverse components of the oval streaming motions must be considered when predicting the expected radial velocity field. However, in the cases of NGC 4618, NGC 4027, and NGC 2537 , it is very clear that the presence of a bar is evident in the ionized gas kinematics. In addition, there is some evidence for systematic departures from circular rotation in the area of the asymmetric arm. Such kinematic features, especially evident in the NGC 2537 field, may be linked to the presence of a stable Lagrangian point associated with the offset stellar bar.

\section{The Frequency of Companion Galaxies}

As discussed by Odewahn (1991), many SBm systems possess close companions, some of which also display the one-armed spiral morphology. In order to assess the likelihood that the asymmetric morphology of the barred Magellanic spirals may be largely due to tidal interactions, a survey of the $\operatorname{largest}\left(\log D_{25}>\right.$ 1.3) Magellanic spiral galaxies in the RC3 catalog was performed using the Palomar and UK Schmidt Sky Surveys. In this analysis (Odewahn 1994), an attempt was made to classify arm strengths and a search for nearby neighbor galaxies was conducted. In a statistical analysis of the data gathered in this survey it was determined that among 75 galaxies with well classified asymmetric arms, only 4 were found to have no nearby neighbor within a separation of $8 \log D_{25}$. The classification of these 4 systems as Magellanic type galaxies is highly questionable (Corwin, private communication). In no case was a bright, dominant arm classified in a system in which a clear neighbor galaxy was absent.

Since very little radial velocity data exists for the neighbor galaxies, indeed few are even included in the RC3, the question of physical association may not be addressed on a system by system basis. It should be noted that in the case of the NGC 4618-4625 system, recent VLA observations presented in Odewahn et al. (1992) clearly confirm that these galaxies comprise a physical pair undergoing strong tidal interaction. A similar study by Phookun et al. (1992) produces the same conclusion for the NGC 4027-4027A system. For the bulk of the galaxies sampled in the current survey, we must approach the question of physical membership, as opposed to chance optical alignment, from a statistical standpoint. Following Holmberg (1969), the frequency distribution of separations for SBm companions, $f(\Delta)$, was compared to various control samples drawn from the RC3 and the deep NGP survey of Odewahn \& Aldering (1995). The sample of one-armed spiral companions (denoted by $\mathrm{Sm}$ in the figure) was divided into three groups to check for effects of sample bias. The first sample consists of the entire set of 77 primary-companion pairs, the second sample is a low redshift set of 35 galaxies with $V \leq 1104 \mathrm{~km} \mathrm{~s}^{-1}$, and the third sample is a high redshift set of 42 galaxies with $V>1104 \mathrm{~km} \mathrm{~s}^{-1}$. The radial velocity used is that of the primary Sm galaxy taken from the RC3. For these three data samples the distributions were computed using equal binning intervals. The cumulative distributions for these $\mathrm{SBm}$ samples are represented by the dashed curves in Figure 2. 


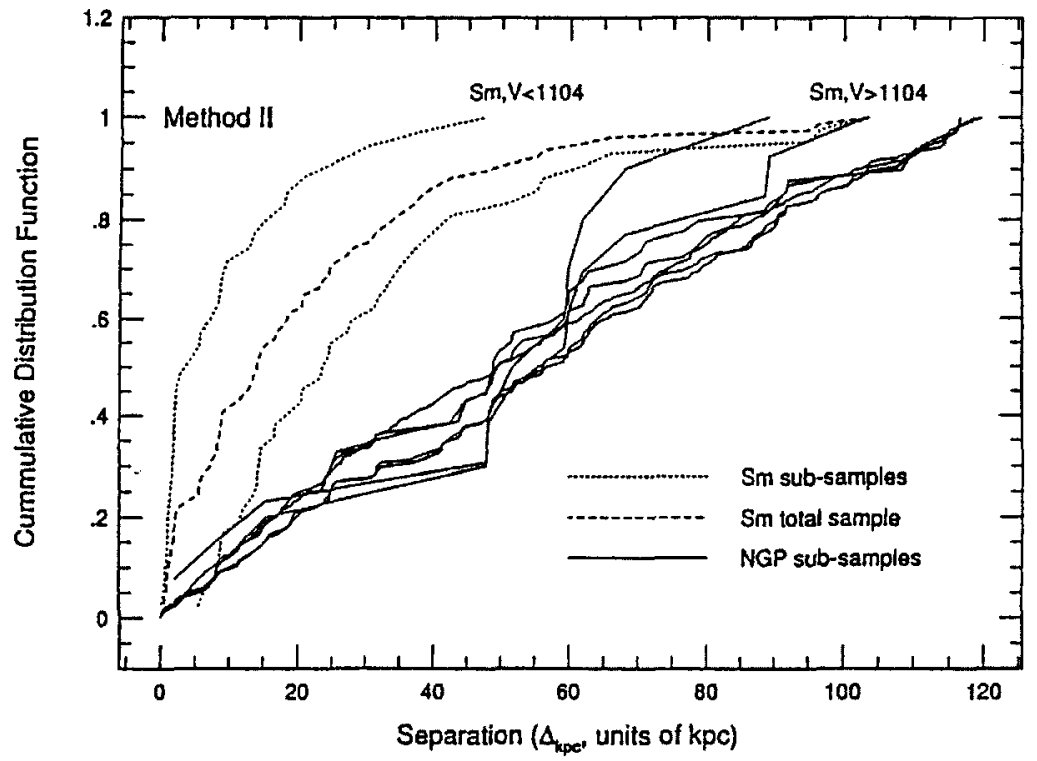

Figure 2. Cumulative distributions of SBm companion separations.

The distributions for $\mathrm{SBm}$ companions rise steeply due to a high relative frequency of close separations. This is directly contrary to what we would expect from chance optical alignments in the case of galaxies homogeneously distributed on the sky.

The assumption of a homogeneous spatial distribution of galaxies is a poor one. In addition, the degree of nonhomogeneity in a given galaxy survey is dependent on morphological type. Hence, we must question whether the relatively large frequency for small Sm companion separations is truly the result of an increased number of physically associated pairs or simply a reflection of the clustered nature of galaxies. In order to evaluate this question a sample of RC3 galaxies covering a range of morphological types and radial velocities has been combined with a deep automated survey of an $18^{\circ} \times 18^{\circ}$ area at the north galactic pole (NGP) using the Automated Plated Scanner (APS) of the University of Minnesota. Subsets of morphological types, with measured radial velocities and isophotal diameters, were selected from the RC3. Using the APS survey, a search was made in the area of each RC3 galaxy for potential companion galaxies out to a radius defined by $10 \log D_{25}$. The resultant catalogs of companion candidates were analyzed in the same fashion as the Sm companion samples. In no case do we find the type of peaked $f(\Delta)$ relation produced by the Sm galaxy companion candidates discussed in this work. A Kolmogorov-Smirnov (KS) test indicates a high probability $(\geq 95 \%)$ that the NGP pole samples (solid lines of Figure 2) are drawn from a parent distribution which is different from that of the Sm samples. 
For several methods of analysis discussed in Odewahn (1994), for a variety of data selection criteria, it is clear that the frequency distribution of separations for Sm galaxy companions is strongly peaked at small separations compared to other subsets. This indicates that the one-armed spiral galaxies $(\mathrm{Sm})$, when compared to other morphological subsets, have a higher probability of having close companion galaxies. This difference would support the view that the pairs sampled in the Sm survey consist predominantly of physical associations as opposed to chance optical alignments. Hence, scenarios invoking the formation of one-armed spiral structures through some tidal interaction mechanism might be attractive, since a common trait among the Magellanic spirals appears to be the presence of a physical neighbor.

Acknowledgments. I thank Dr. Gerard de Vaucouleurs for many useful and enjoyable discussions concerning the photometry and dynamics of late-type galaxies.

\section{References}

Binney, J. 1981, MNRAS, 196, 455

Bothun, G. \& Thompson, I. 1988, AJ, 96, 877

de Vaucouleurs, G. 1959, Handbuch der Physik, 53, 275

de Vaucouleurs, G. \& Freeman, K. C. 1973, Vistas in Astronomy, 14, 163

de Vaucouleurs, G., de Vaucouleurs, A., Corwin, H. G. Jr., Buta, R. J., Paturel, G., \& Fouqué, P. 1991, Third Reference Catalogue of Bright Galaxies, New York: Springer

Duus, A. \& Freeman, K. C. 1975, in La Dynamique des Galaxies Spirales, L. Weliachew, Paris: Centre National de la Recherche Scientifique, 419

Elmegreen, B. \& Elmegreen, D. 1985, ApJ, 288, 438

Feitzinger, J. 1980, Space Science Review, 27, 35

Holmberg, E. 1969, Arkiv Astr., 5, 305

Odewahn, S. C. 1989, Properties of the Magellanic Type Galaxies, Ph.D. Dissertation, University of Texas

Odewahn, S. C. 1991, AJ, 101, 829

Odewahn, S. C. 1994, AJ, 107, 1320

Odewahn, S. C. \& Aldering, G. 1995, AJ, 110, 2009

Odewahn, S. C., Woodward, C. E., \& Bailey, J. 1992, in Proceedings of the Third Teton Summer School on Astrophysics: The Evolution of Galaxies and Their Environment, D. Hollenbach, H. Thronson, \& J. M. Shull, NASA Conference Publication 3190, 235

Pence, W. D., Taylor, K., Freeman, K. C., de Vaucouleurs, G., \& Atherton, P. 1988, ApJ, 326, 564

Phookun, B., Mundy, L. G., Teuben, P. J., \& Wainscoat, R. J. 1992, ApJ, 400, 516 